\title{
Roughness effect on the efficiency of dimer antenna based biosensor
}

\author{
Sameh Kessentini, Dominique Barchiesi \\ Automatic Mesh Generation and Advanced Methods (GAMMA3 UTT/INRIA) \\ University of Technology of Troyes \\ 12 rue Marie Curie - CS 42060 - FR-10004 Troyes cedex \\ Corresponding author, E-mail: dominique.barchiesi@utt.fr, SamehKessentini@gmail.com
}

\begin{abstract}
The fabrication process of nanodevices is continually improved. However, most of the nanodevices, such as biosensors present rough surfaces with mean roughness of some nanometers even if the deposition rate of material is better and better controlled. The effect of roughness on performance of biosensors was fully addressed for plane biosensors and gratings, but rarely addressed for biosensors based on Local Plasmon Resonance. The purpose of this paper is to evaluate numerically the influence of nanometric roughness on the efficiency of a dimer nano-biosensor (two levels of roughness are considered). Therefore, we propose a general numerical method, that can be applied to any other nanometric shape, to take into account the roughness in a three dimensional model. The study focuses on both the far-field, which corresponds to the experimental detected data, and the near-field, responsible for exciting and then detecting biological molecules. The results suggest that the biosensor efficiency is highly sensitive to the surface roughness. The roughness can produce important shifts of the extinction efficiency peak and a decrease of its amplitude resulting from changes in the distribution of near-field and absorbed electric field intensities.
\end{abstract}

\section{Introduction}

During the last years the fabrication process of nanodevices has been continuously improved and more control and precision were achieved [1]. However, most of the nanodevices, such as biosensors present rough surfaces with mean roughness of some nanometers. The effect of roughness on performance of biosensors was fully addressed for plane biosensors or gratings (mainly the angular dependence) [2-6] but rarely for the different new shapes of biosensors [7]. Moreover, for the new shapes, the few studies reported are experimental and no modeling work was done. One of the few studies addressing the roughness effect on new shapes of biosensor is the one done by Reilly et al. in 2007 [8]. In that study, authors claim that roughness enhances the SERS (Surface Enhanced Raman Spectroscopy) signal as the calculated enhancement factor on smooth structure is lower than the experimentally measured one with rough surface. However the difference between experimental and numerical calculation may result from other factors such as the choice of optical index of metal, the precision of the numerical method, the fabrication er- rors, etc. Therefore, a theoretical comparison of smooth and rough surfaces (with different level of roughness) is critical to get more significant conclusions.

Few modeling studies took account of the biosensor roughness. Michel et al. [5] considered a roughened grating composed of the sum of a sinusoidal profile and a onedimensional Gaussian random process. Byun et al. $[4,5]$ modeled the roughness using one-dimensional Gaussian random surface of plan biosensor or gratings. All these previous studies [4-6] are restricted to specific biosensors or particles [7] and sometimes present one-dimensional model quite different from the three dimensional one needed to fully describe rough surfaces of biosensors. To overcome the limitations of these models, a numerical method that can describe rough surface should be used (the analytical solution being known only for simple shapes of biosensors). Some numerical methods can deal with different shapes but most of them necessitate fitting boundary conditions which makes describing a rough surface critical [7]. Hence, we decide to use the discrete dipole approximation (DDA) which is volume-integral method overcoming this limitation. Finally, using the DDA, the rough surface can be easily described by adding or removing material from the target surface as it be will fully explained in the second section.

The model is applied to a dimer antenna based biosensor. Two characteristics of the biosensor should be improved for SERS (Surface Enhanced Raman Spectroscopy) applications. Firstly, the position of the plasmon resonance, described by the spectroscopic model in far field, should be adjusted to match with target biomolecule characteristics in SERS. Secondly, the field intensity in the vicinity of the biosensor should be enough to yield intense SERS signal from molecules enabling their identification. To deal with both issues, we analyze the effect of roughness on the dimer antenna based biosensor in the far-field and near-field as well as the correlation between the two levels.

This paper is organized as follows: the second section present an overview of the DDA and describe the method used to model the rough surface. The third section is devoted to the validation of the model by comparison with experimental results, and the fourth one gives different simulation results on nanoantenna and their discussion. Finally conclusions and future works will be presented in the fifth section. 


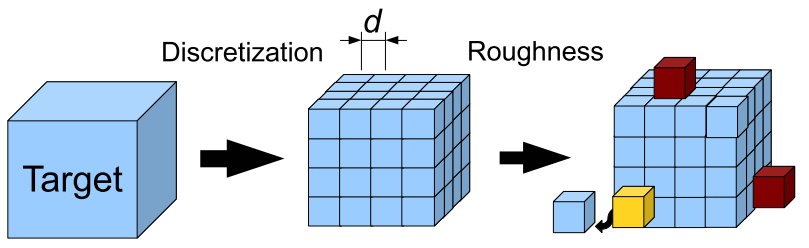

Figure 1: The basic idea of DDA: discretizing the target in a set of dipoles, and roughness control.

\section{Numerical method and model of rough surface}

Maxwell's equations describe the interaction of biosensor with incoming illumination. When the analytical solutions of these equations are unknown, it is necessary to use numerical methods. A number of numerical methods were introduced such as the discrete dipole approximation (DDA), the method of moments, the finite difference time domain method and the finite element method. Each of these methods presents some advantages and drawbacks. Some of them are able to deal with $3 \mathrm{D}$ target shapes with smooth surfaces. However, including roughnesses can be critical $[4,7,9-11]$. In fact, to get a three-dimensional rough object, small pieces of materials should be added to or removed from the surface. Therefore, the numerical methods that necessitate setting the boundary conditions at surfaces (unlike DDA) are less suitable.

The DDA was firstly developed by Devoe $[12,13]$ and Purcell and Pennypacker [14]. The accuracy of DDA was proved when compared to exact solutions for some specific shapes (spherical nanoparticle [15], ellipsoid [15] and infinite cylinder [16]). The main idea of DDA is to discretize a target shape into a set of $N$ polarizable elements (figure 1). These elements get dipole moments and interact each with others when an electric field is applied and hence are called dipoles. Details on the theoretical setting of the method can be found in many papers such as [17]; but here we concentrate on how the method can be investigated to model rough surface of any target shape. Many development of the method were done $[16,18,19]$ to deal with periodic structures. These latest contributions make the DDA method able to compute the scattering and absorption of biosensors based on reproducing periodically a shape as the dimer antenna based biosensor considered in this study (figure 2). Due to the dipolar meshing of objects, DDA is able to describe any biosensor with arbitrary shape and even with rough surface, with minimal efforts .

The absorption, scattering or extinction efficiencies as well as electromagnetic field could be computed using DDSCAT 7.1, which is a Fortran code based on the DDA [20,21]. This code can be edited to change some of the existing shapes or introducing new ones. Therefore, we first introduce the smooth target dimer antenna presented by figure 2 including the adhesion layer (usually ignored in the modeling work but of great influence $[22,23]$. Then, a modified version of this shape is introduced by adding or

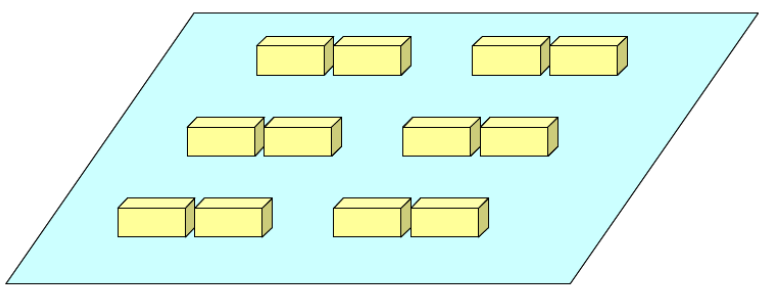

Figure 2: Nanonantenna based biosensor: two-dimensional array of dimer antenna.

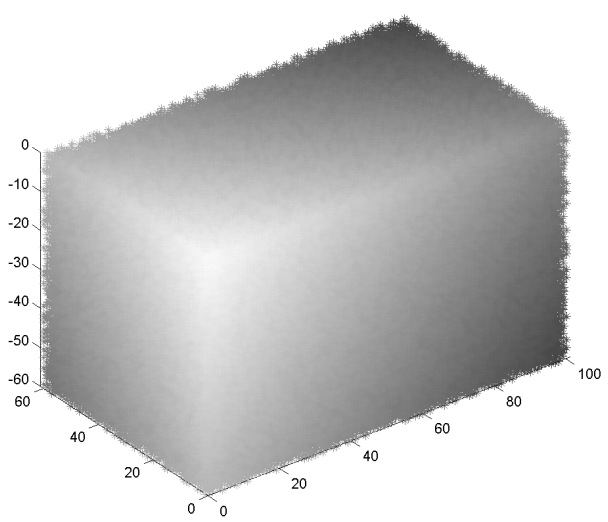

Figure 3: Nanorod with rough surface. Each dipole is represented by a star.

removing dipoles to/from the surfaces to get the rough object [24]. We should note that different level of roughness can be simulated by removing or adding more than a dipole at once (see Fig. 1). The root-mean-square $(\mathrm{rms})$ roughness is controlled through a uniform probability law governing the events of adding, removing or keeping dipoles at the surface of objects. Figure 3 shows an example of rough nanoantenna (the surface presents some granulations).

In the following section we propose a validation of the model by using a comparison of the results with or without roughness with those of experiments.

\section{Validation of the model}

The lack of systematic experimental studies of the above described nanoantennas by varying sizes for example, force us to turn to cylindrical shapes. Two validations are proposed, using experimental results from [25, Fig. 2] and [26].

In that references the extinction spectrum of cylindrical gold nanostructures was measured. The maximum of $Q_{\text {ext }}$ over the wavelength $\lambda_{0}$ is associated to the Localized Surface Plasmon Resonance (LSPR) [27]: $\max _{\lambda_{0}}\left(Q_{\text {ext }}\right)$. In experiments, the uncertainty on the determination of the wavelength corresponding to this maximum is evaluated to $\pm 20 \mathrm{~nm}$ [28]. The Scanning Electron Microscope images 


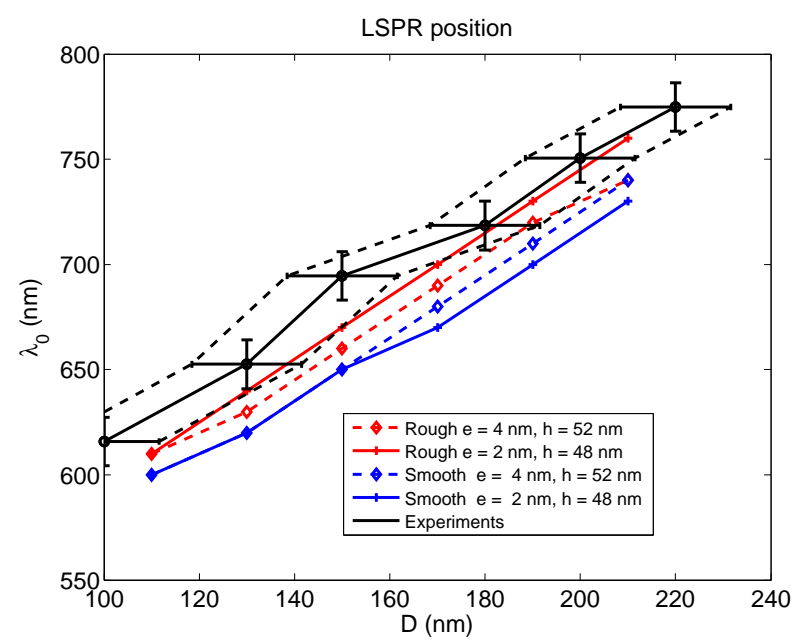

Figure 4: Experimental data from [25] with standard uncertainties. Intervals for the position of the LSPR computed with DDA, using experimental uncertainties, for smooth and rough nanostructures.

show residual roughness. The smooth and rough samples are considered for the model. The distance between samples is $P=200 \mathrm{~nm}$. A chromium adhesion layer with thickness of $e=3 \pm 1 \mathrm{~nm}$ was used to stick gold structures on the $\mathrm{CaF}_{2}$ substrate. The maximum uncertainty on height of nanostructures was $\pm 2 \mathrm{~nm}$, that on diameter was $\pm 20 \mathrm{~nm}$. The sizes are supposed to be uniformly distributed in the above intervals. Then the standard uncertainty are the ratio of the above maximum uncertainties to $\sqrt{3}$ [29]. These values are reported as error bars for the experimental data in Figs. 4-5.

For the validation, we consider the maximum experimental uncertainties on sizes to generate numerical data. Actually it is well known that increasing the diameter $D$ or decreasing the height $h$ of cylinders redshift the LSPR [30]. The influence of the adhesion layer for $D=100 \mathrm{~nm}$ is a tiny blueshift [31]. Therefore, intervals for the positions of the LSPR can be numerically evaluated by using the experimental uncertainties. In Figs. 4-5, the computed LSPR falls between the dashed and the solid lines for smooth (blue) and rough (red) structures.

Figure 4 shows the result a systematic study of the diameter $D$ of cylinders, their height being fixed to $50 \mathrm{~nm}$. For figure 5, the diameter is fixed to $100 \mathrm{~nm}$ and the height of dots is varied. Both figures show a better agreement between rough numerical results and experimental data. The intersection of the intervals of uncertainty is visible for rough structures on the contrary of smooth ones.

For $D=100 \mathrm{~nm}, h=50 \mathrm{~nm}, P=200 \mathrm{~nm}$, DDA gives results $\left(\lambda_{0}(L S P R) \in[603 ; 628]\right)$ that are closer to experiments [25] $\left(\lambda_{0}(L S P R)=615 \mathrm{~nm}\right)$ than FDTD [31], where the LSPR was found between $541 \mathrm{~nm}(e=5 \mathrm{~nm})$ and $583 \mathrm{~nm}(e=1 \mathrm{~nm})$

For each diameter $D$ (Fig. 4) or height $h$ (Fig. 5), the difference of LSPR between the dashed and solid lines

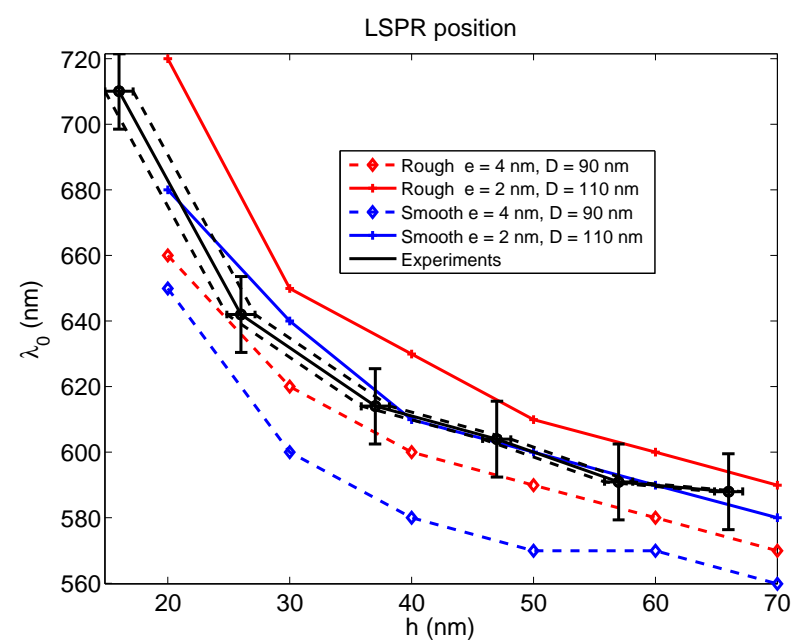

Figure 5: Experimental data from [26] with standard uncertainties. Intervals for the position of the LSPR computed with DDA, using experimental uncertainties, for smooth and rough nanostructures.

can be considered as an evaluation of the sensitivity of the LSPR to the uncertainty on the thickness of chromium and on the size of structures. The sensitivity is lower than $10 \mathrm{~nm}$ for all diameters and is almost constant. On the contrary, the sensitivity on $h$ for $D=100 \mathrm{~nm}$ decreases with $h$. The agreement between rough simulations and experiments is better for significant heights and the experimental uncertainty for small heights may be undervalued.

The comparisons of numerical results to experimental data show that the rough model with adhesion layer is more efficient to describe the experiments on a wide range of parameters. Therefore, the proposed model may be extended to the nanoantenna shapes.

\section{Numerical simulations and discussion}

We consider the biosensors represented in figure 2 with the following size characteristics: each of the nanorods has length of $100 \mathrm{~nm}$ (along $\mathrm{x}$-axis), and width and height of $60 \mathrm{~nm}$. A gap of $20 \mathrm{~nm}$ separates the pairs that are reproduced periodically with a separation distance of $200 \mathrm{~nm}$. For all simulations we use Johnson and Christy optical index for gold and choose an inter-dipole distance of $1 \mathrm{~nm}$ (which is also the size parameter of a dipole). Then, we apply the procedure described in the previous section to get rough surface by adding or removing a unique dipole in a first case and up to two dipoles in a second case. The used random variable is based on uniform probability law, so that the events of adding, removing or keeping the same height are equiprobable. Hence, the rootmean-square $(\mathrm{rms})$ surface height deviation from a mean plane are $r m s=\sqrt{\left((-1)^{2}+0+1^{2}\right) / 3}=0.82 \mathrm{~nm}$ and $r m s=\sqrt{\left((-2)^{2}+(-1)^{2}+0+1^{2}+2^{2}\right) / 5}=1.41 \mathrm{~nm}$, respectively.

We present the effect of surface roughness on the LSPR 


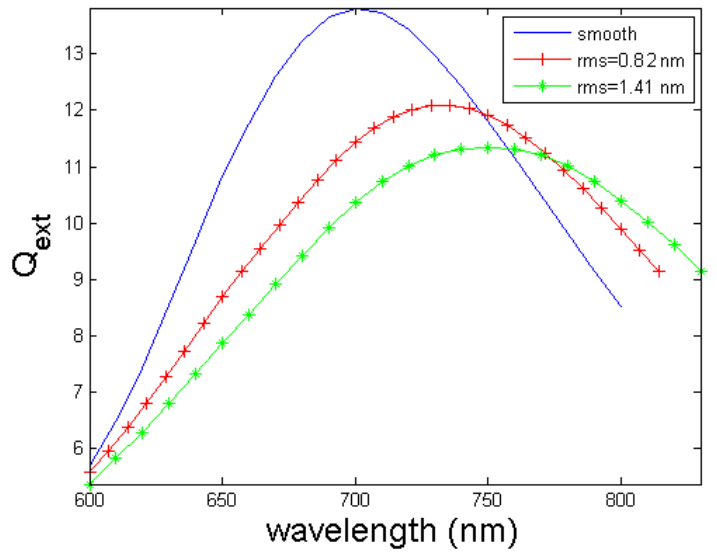

Figure 6: Extinction efficiency spectra of nanoantennabased biosensor for smooth (blue curves) and rough topography.

biosensor characteristics. Therefore we plot the extinction, absorption and scattering spectra as a function of the wavelength for smooth and rough surfaces. The comparison will be held in terms of extinction peak wavelength and amplitude as well as the absorption and scattering efficiencies. The roughness deteriorates the scattering peak amplitude while the absorption peak amplitude is improved as shown by figures 7-8. For rougher surface, the overall extinction peak amplitude decreases as the drop of scattering is more drastic than the increase of absorption. Therefore, to get a maximal extinction peak amplitude the surface of the biosensor should be as smooth as possible. Finally, for the considered biosensor, the roughness red shifts the wavelength of extinction peak (the shift is about 50 $\mathrm{nm}$ when comparing the results for the rough structure of $r m s=1.41 \mathrm{~nm}$ to the smooth one). The redshift of the extinction peak is greater for nanoantenna than for cylinder (section 3). This behavior is probably due to the roughness of the nanometric gap for nanoantenna where the field enhancement is known to be very intense and confined.

As a first conclusion, these results suggest that the effect of roughness on biosensor efficiency could not be ignored. This is expected as the considered biosensor size parameters are some tens of nanometers and hence a surface with roughness of some nanometers cannot be considered as smooth. The roughness effects are basically:

- A red shift of the extinction peak (figure 6). The redshift of LSPR is also observed for cylindrical nanostructures in section 3 .

- A drop of the scattering and extinction peak amplitude (figures 6 and 8).

- An increase of the absorption peak amplitude (figure 7).

As commonly thought, the roughness is supposed to produce efficient nanosources, which could excite locally

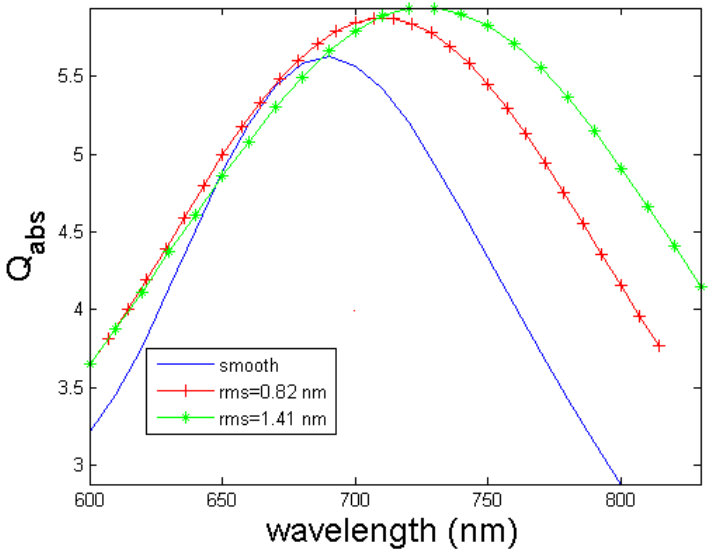

Figure 7: Absorption efficiency spectra of nanoantennabased biosensor for smooth (blue curves) and rough topography.

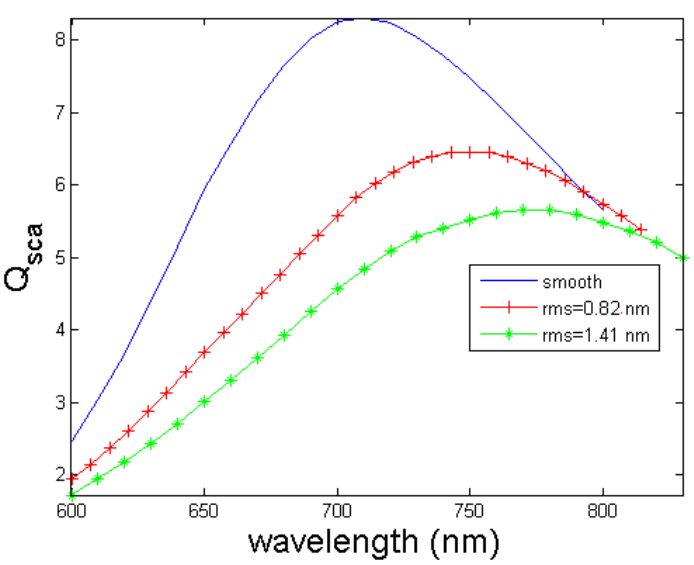

Figure 8: Scattering efficiency spectra of nanoantennabased biosensor for smooth (blue curves) and rough topography.

the target molecules. The impact of rough surface on SPR characteristics of plane biosensor was already studied by Raether [2]. Raether found that for small roughness, in the range of $0.5-2 \mathrm{~nm}$, the nonradiative surface plasmons (SPs) become radiative as a result of the optical coupling between excited SPs and photons. Hence, rough nanoantennas are expected to be more diffusive objects, however we find that their scattering efficiencies are less than those of the smooth nanoantennas (figure 8). The reasons of such behavior should be clarified. Actually, prediction of behavior of the nanosensor is difficult as the absorption is in competition with the scattering and more analysis is needed.

The above mentioned far field quantities are the unique measurable values in experimental setup. However, the electric field intensity $|E|^{2}$ just behind the surface is the source of the far field quantities. It is also more interesting to characterize the biosensor ability to excite biological molecules trapped at few nanometers from its surface. 
Therefore to depict the effect of roughness on electric field absorbed and diffracted parts, respectively, we compute the intensity in the symmetry plane along the two nanorods at $3 \mathrm{~nm}$ respectively below and above the mean upper surface for a wavelength of $720 \mathrm{~nm}$ (figures 9-10).

The electric field intensity $|E|^{2}$ inside the biosensor and in the gap is locally enhanced for rough surfaces (red and green curves in figure 9). This enhancement contributes to the difference in the spectra of the absorption efficiency (figure. 7). On the other hand, the influence of roughness on the electric field intensity above the biosensor (figure 10) seems to be less important than inside it. The mean value of the near-field intensity is equal to $23.7,22.8$ and 27.7 for smooth surface, rough surfaces with $r m s=0.81$ and $r m s=1.41$, respectively. This shows that the contribution of the smallest roughnesses $(r m s=0.81)$ decreases more quickly when getting a few nanometers far from the surface. However, when computing the electric field intensity, $6 \mathrm{~nm}$ away from the mean surface (the typical position of the biomolecules stuck on a functionalization layer deposited on the metallic part of biosensor), we could conclude that the roughest the surface is, the less the intensity is (figure 11). The near-field depends on not only to the distance (different levels can be seen in figures 10-11) and the roughness level, but also to the absorption of light by the gold nanorods. The near-field intensity is more intense for the roughest biosensor, just above the surface (figure 10), but not a few nanometers far from the surface. Therefore, the overall effect is actually a decrease of the scattering efficiency as shown in figure 8.

As a general conclusion, simulations show a competition between two physical effects induced by roughness (increase of absorption and decrease of scattering) and a local enhancement of field damped rapidly with distance, yielding important changes in the behavior of the considered biosensor.

\section{Conclusion}

In this study, we modeled rough surface to investigate the effect of roughness on the efficiency of nanoantenna-based biosensor. The model is based on using the DDA method and randomly adding or removing material to/from the surface. A validation of the model by comparison with experimental data over a range of size parameters is given by taking into account the experimental uncertainties. This comparison shows that the model including roughness is more efficient to describe experiments. This model can be applied to any other nanometric sample and is more suitable than models suggested as previous studies [32,33] as it reproduces the effective three dimensional rough surface as in [7]. The model is applied to nanoantenna considering smooth surface and two levels of roughnesses. The results suggest that the biosensor efficiency is highly sensitive to the surface roughness: the extinction efficiency peak decreases with roughness and its position (wavelength) is red-shifted. These effects are related to alteration in local absorption and scattering of the electric field. In fact, the

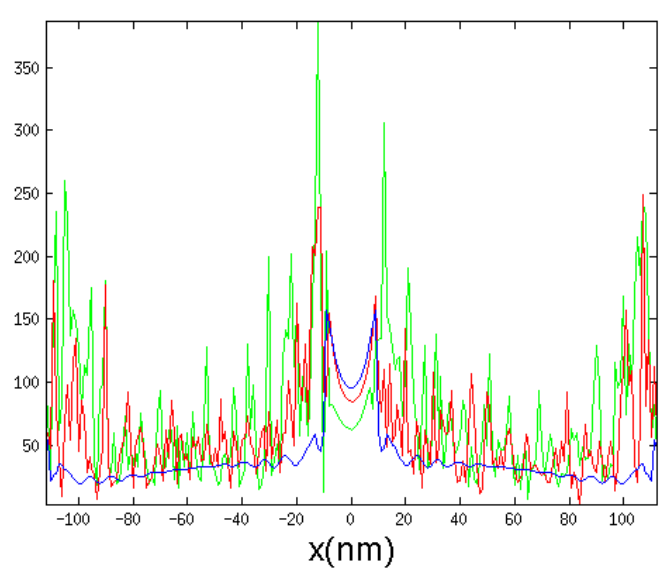

Figure 9: Intensity of the electric field $|E|^{2}$ inside the nanoantenna (-: biosensor with smooth surface, - : biosensor with rms roughness $0.82 \mathrm{~nm}$ and - - biosensor with rms roughness $1.41 \mathrm{~nm}$; the gap between the two nanorods is from $x=-10$ to $x=10 \mathrm{~nm}$ )

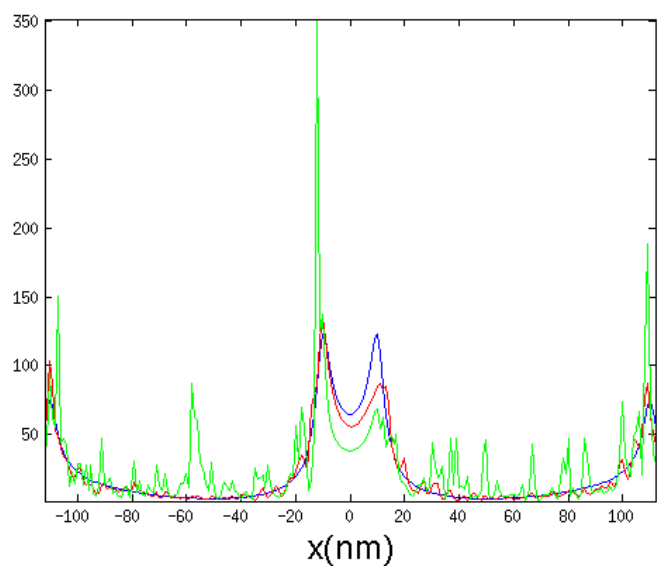

Figure 10: Intensity of the electric field $|E|^{2}$ at $3 \mathrm{~nm}$ above the nanoantenna ( - : biosensor with smooth surface, - :

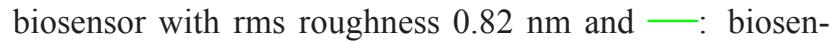
sor with rms roughness $1.41 \mathrm{~nm}$; the gap between the two nanorods is from $x=-10$ to $x=10 \mathrm{~nm}$ )

roughness disturbed the distribution of electric field intensity both inside and outside the biosensor as shown by simulations. The results obtained here may be related to the considered biosensor and roughness levels (e.g. red or blue shift of extinction peak). However, a general conclusion can be made: the roughness could not be ignored in modeling studies as the more the surface is rough the more the distribution of electric field is disturbed [34]. We join Reilly et al. [8] when stating that difference between experimental and numerical studies may result in part from roughness. In future works, this model will be applied to some specific shapes of biosensors of given roughness as we expect a better match with experimental data and a better reliability for their optimization [35-37]. 


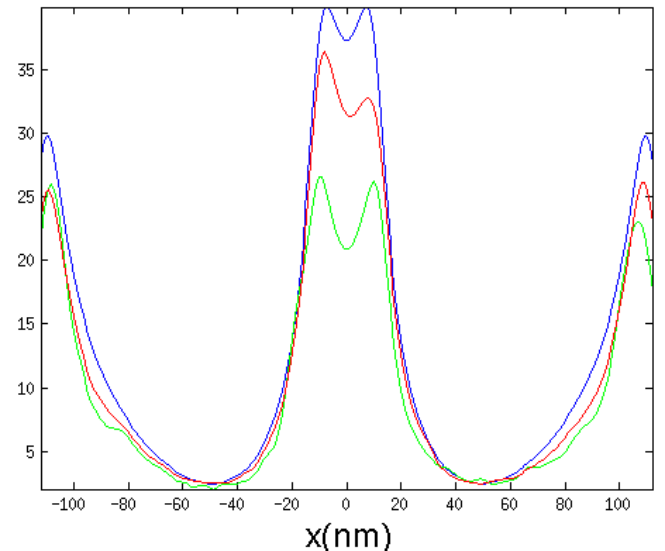

Figure 11: Intensity of the electric field $|E|^{2}$ at $6 \mathrm{~nm}$ above the nanoantenna (- biosensor with smooth surface, - : biosensor with rms roughness $0.82 \mathrm{~nm}$ and - - biosensor with rms roughness $1.41 \mathrm{~nm}$; the gap between the two nanorods is from $x=-10$ to $x=10 \mathrm{~nm}$ )

\section{Acknowledgement}

Authors are grateful to Marc Lamy de la Chapelle and

Nicolas Guillot for providing experimental data previously published in [25]. This work was supported by the "Conseil Régional de Champagne Ardenne", the "Conseil Général de l'Aube" and the Nanoantenna European Project (FP7 Health-F5-2009-241818).

\section{References}

[1] A. A. Yanik, M. Huang, A. Artar, T.-Y. Chang, and H. Altug, "On-chip nanoplasmonic biosensors with actively controlled nanofluidic surface delivery," in Plasmonics: Metallic Nanostructures and Their Optical Properties VIII, M. I. Stockman, Ed., vol. 7757. San Diego, California, USA: SPIE, August 2010, pp. 775 735-1-6.

[2] H. Raether, Surface Plasmons on Smooth and Rough Surfaces and on Gratings. Berlin: Springer-Verlag, 1988.

[3] E. Kretschmann, "The angular dependence and the polarisation of light emitted by surface plasmons on metals due to roughness," Optics Communications, vol. 5, no. 5, pp. 331-336, 1972.

[4] K. M. Byun, S. J. Yoon, D. Kim, and S. J. Kim, "Sensitivity analysis of a nanowire-based surface plasmon resonance biosensor in the presence of surface roughness," J. Opt. Soc. Am. A, vol. 24, no. 2, pp. 522-529, 2007.

[5] T. R. Michel, M. E. Knotts, and K. A. O'Donnell, "Scattering by plasmon polaritons on a rough surface with a periodic component," J. Opt. Soc. Am. A, vol. 12 , no. 3 , pp. 548-559, 1995.
[6] K. M. Byun, S. J. Yoon, and D. Kim, "Effect of surface roughness on the extinction-based localized surface plasmon resonance biosensor," Appl. Opt., vol. 47, no. 1, pp. 5886-5892, 2008.

[7] A. Trügler, J.-C. Tinguely, J. R. Krenn, A. Hohenau, and U. Hohenester, "Influence of surface roughness on the optical properties of plasmonic nanoparticles," Phys. Rev. B, vol. 83, pp. 081 412-1-081412-4, 2011.

[8] T. H. Reilly, S. H. Chang, J. D. C. G. C. Schatz, and K. L. Rowlen, "Quantitive evaluation of plasmon enhanced Raman scattering from nanoaperture arrays," J. Phys. Chem. C, vol. 111, pp. 1689-1694, 2007.

[9] F. D. Hastings, J. B. Schneider, and S. L. Broschat, "A Monte-Carlo FDTD technique for rough surface scattering," IEEE Transactions on antennas and propagation, vol. 43, no. 11, pp. 1183-1191, 1995.

[10] V. Poroshin, Y. Borovin, and D. Bogomolov, "Transfer of the surface roughness geometry into the universal FEM software ANSYS," Advanced Engineering, vol. 3, no. 2, pp. 1846-5900, 2009.

[11] A. Kato, S. Burger, and F. Scholze, "Analytical modeling and three-dimensional finite element simulation in line edge roughness in scatterometry," Appl. Opt., vol. 51, no. 27, pp. 6457-6464, 2012.

[12] H. Devoe, "Optical properties of molecular aggregates. I. classical model of electronic absorption and refraction," J. Chem. Phys., vol. 41, pp. 393-400, 1964.

[13] — , "Optical properties of molecular aggregates. II. classical theory of the refraction, absorption, and optical activity of solutions and crystals," J. Chem. Phys., vol. 43, pp. 3199-3208, 1965.

[14] E. Purcell and C. R. Pennypacker, "Scattering and absorption of light by nonspherical dielectric grains," Astrophysical Journal, vol. 186, pp. 705-714, 1973.

[15] K. S. Lee and M. A. El-Sayed, "Dependence of the enhanced optical scattering efficiency relative to that of absorption of gold metal nanorods on aspect ratio, size, end-cap shape, and medium refractive," J. Phys. Chem. B, vol. 109, pp. 20331-20338, 2005.

[16] B. T. Draine and P. J. Flatau, "Discrete-dipole approximation for periodic targets: Theory and tests," J. Opt. Soc. Am. A, vol. 25, pp. 2693-2703, 2008.

[17] B. T. Draine, "The discrete dipole approximation and its application to interstellar graphite grains," The Astrophysical Journal, vol. 333, pp. 848-872, 1988.

[18] V. A. Markel, "Coupled-dipole approach to scattering of light from a one-dimensional periodic dipole structure," J. Mod. Opt., vol. 40, no. 11, pp. 2281-2291, 1993. 
[19] P. C. Chaumet, A. Rahmani, and G. W. Bryant, "Generalization of the coupled dipole method to periodic structures," Phys. Rev. B, vol. 67, no. 16, pp. $165404(1-5), 2003$.

[20] B. T. Draine and P. J. Flatau, "User guide to the discrete dipole approximation code DDSCAT 7.1," http://arXiv.org/abs/1002.1505v1, 2010.

[21] — , "Discrete-dipole approximation for scattering calculations," J. Opt. Soc. Am. A, vol. 11, pp. 14911499, 1994.

[22] D. Barchiesi, D. Macías, L. Belmar-Letellier, D. Van Labeke, M. Lamy de la Chapelle, T. Toury, E. Kremer, L. Moreau, and T. Grosges, "Plasmonics: Influence of the intermediate (or stick) layer on the efficiency of sensors," Appl. Phys. B, vol. 93, pp. 177-181, 2008.

[23] Y. B. Zheng, B. K. Juluri, X. Mao, T. R. Walker, and T. J. Huang, "Systematic investigation of localized surface plasmon resonance of long-range ordered $\mathrm{Au}$ nanodisk arrays," J. Appl. Phys, vol. 103, pp. 014308 1-014308-9, 2008.

[24] M. J. Banholzer, S. Li, J. B. Ketter, D. I. Rozkiewicz, G. C. Schatz, and C. A. Mirkin, "An electrochemical approach to and the physical consequences of preparing nanostructures from gold nanorods with smooth ends," J. Phys. Chem. C Nanomater Interfaces, vol. 112, no. 40, pp. 15 729-15734, 2008.

[25] N. Guillot, , H. Shen, B. Frémaux, O. Péron, E. Rinnert, T. Toury, and M. Lamy de la Chapelle, "Surface enhanced Raman scattering optimization of gold nanocylinder arrays: Influence of the localized surface plasmon resonance and excitation wavelength," Appl. Phys. Lett., vol. 97, no. 2, pp. 023 113-023 116,, 2010.

[26] J. Grand, "Plasmons de surface de nanoparticules : spectroscopie d'extinction en champs proche et lointain, diffusion Raman exaltée," Ph.D. dissertation, Université de technologie de Troyes, 2004.

[27] E. C. Le Ru and P. G. Etchegoin, Principles of Surface-Enhanced Raman Spectroscopy and related plasmonic effects, 1st ed. Amsterdam: Elsevier, 2009.

[28] N. Félidj, J. Aubard, G. Lévi, J. R. Krenn, M. Salerno, G. Schider, B. Lamprecht, A. Leitner, and F. R. Aussenegg, "Controlling the optical response of regular arrays of gold particles for surface-enhanced Raman scattering," Phys. Rev. B, vol. 65, pp. 075419 $075427,2002$.

[29] Working Group 1, Evaluation of measurement data - Guide to the expression of uncertainty in measurement, 1st ed., Joint Committee for Guides in Metrology, Paris, 2008, corrected version 2010.
[30] M. Pelton, J. Aizpurua, and G. W. Bryant, "Metalnanoparticles plasmonics," Laser \& Photon. Rev. vol. 2, no. 3, pp. 136-159, 2008.

[31] A. Vial and T. Laroche, "Description of dispersion properties of metals by means of the critical points model and application to the study of resonant structures using the FDTD method," J. Phys. D: Appl. Phys., vol. 40, pp. 7152-7158, 2007.

[32] B. Guizal, D. Barchiesi, and D. Felbacq, "Electromagnetic beam diffraction by a finite lamellar structure," J. Opt. Soc. Am. A, vol. 20, no. 12, pp. 2274-2280, 2003.

[33] R. Fikri, T. Grosges, and D. Barchiesi, "Apertureless scanning near-field optical microscopy : On the need of the tip vibration modelling," Opt. Lett., vol. 28, no. 22, pp. 2147-2149, 2003.

[34] G. Parent, D. Van Labeke, and D. Barchiesi, "Fluorescence lifetime of a molecule near a corrugated interface. application to near-field microscopy," J. Opt. Soc. Am. A, vol. 16, pp. 896-908, 1999.

[35] D. Macías, A. Vial, and D. Barchiesi, "Application of evolution strategies for the solution of an inverse problem in Near-Field Optics," J. Opt. Soc. Am. A, vol. 21, no. 8, pp. 1465-1471, 2004.

[36] T. Grosges, D. Barchiesi, T. Toury, and G. Gréhan, "Design of nanostructures for imaging and biomedical applications by plasmonic optimization," Opt. Lett., vol. 33, no. 23, pp. 2812-2814, 2008.

[37] S. Kessentini, D. Barchiesi, T. Grosges, and M. Lamy de la Chapelle, "Particle swarm optimization and evolutionary methods for plasmonic biomedical applications," in IEEE Congress on Evolutionary Computation (CEC 2011), IEEE. New Orleans: IEEE, 2010, pp. 2315-2320. 